ISSN electrónico: 2172-9077

DOI: https://doi.org/10.14201/fjc2016135564

\title{
LA PANTALLA DE CINE ABANDONA LA SALA
}

\section{The Cinema Screen Leaves the Theatre}

\author{
David VICENTE TORRICO \\ Personal Investigador en Formación - Universidad de Valladolid \\ E-mail: david.vicente.torrico@uva.es \\ (D) http://orcid.org/0000-0003-0379-6086
}

Fecha de recepción del artículo: 30/09/2016

Fecha de aceptación definitiva: 13/10/2016

\begin{abstract}
RESUMEN
La exhibición cinematográfica, un negocio que el año pasado recaudó 571 millones de euros y congregó en las salas a más de 94 millones de espectadores, no puede mantenerse al margen de la revolución que supone la cuarta pantalla, es decir, la telefonía móvil. En un mercado dominado por dos grandes empresas, Cinesa y Yelmo, planteamos un estudio cualitativo de sus aplicaciones digitales, atendiendo a cuestiones como el diseño, la accesibilidad y sus potencialidades, con el objetivo de comprobar su adaptación a este nuevo entorno digital. Los resultados demostrarán una incipiente apertura, si bien son múltiples las prestaciones que se deberán implementar para aportar un valor añadido al espectador cinematográfico.
\end{abstract}

Palabras clave: Cuarta pantalla; Exhibición; Cine; App; Yelmo; Cinesa.

\begin{abstract}
Last year, the cinematographic exhibition grossed 571 million Euros and congregated more than 94 million spectators. Nowadays, however, this business cannot stay at the edge of the fourth screen's revolution, that is to say, mobile phones. In a market controlled by two big enterprises, namely Cinesa and Yelmo, we propose a qualitative study of its digital applications, considering their design, their accessibility and their features, with the goal of verifying their adaptation to this new digital environment. The results will show an emerging opening. Nevertheless, numerous services must be implemented to enhance the spectator's cinematographic experience.
\end{abstract}

Key words: Fourth screen; Exhibition; Cinema; App, Yelmo; Cinesa. 


\section{INTRODUCCIÓN Y JUSTIFICACIÓN DE LA INVESTIGACIÓN}

La industria audiovisual afronta en la actualidad la enésima revolución tecnológica, aunque en esta ocasión existe una clara particularidad, y es que la batalla se libra de puertas para afuera, en el ámbito digital. El imparable acceso al terreno de juego de un dispositivo, el teléfono inteligente (en adelante, smartphone) -y en menor medida la tablet-, reconfigura no solo el ámbito, sino también la forma de consumo de contenidos audiovisuales, dando lugar a lo que conocemos como la cuarta pantalla, un nuevo espacio caracterizado por la ubicuidad, la inmediatez, la personalización y la interactividad.

\subsection{EL SMARTPHONE}

El smartphone, tal y como señala Costa Sánchez $(2012,2014)$ aúna dos cualidades básicas que le han permitido coronarse como el dispositivo multimedia por excelencia, que son la movilidad y la conectividad a la red. Lejos quedan, por tanto, los tiempos en los que su función estaba limitada a las llamadas y los mensajes de texto, y es que, sin perder de vista esta concepción primigenia, los actuales dispositivos han adquirido nuevos usos, como el de informarse, trabajar o entretenerse, revolucionando por completo el mercado de creación de contenidos digitales.

Su avanzada tecnología ha propiciado que, desde el año 2007 (fecha de lanzamiento del reinventado concepto de smartphone, el iPhone de Apple), estos ordenadores en miniatura hayan alcanzado un índice de penetración del 87\% en España, lo que sitúa al país como líder a nivel europeo, según señala la última publicación de la consultora Ditrendia, elaborada a finales de julio de 2016.

Los datos hablan por sí solos. El mismo estudio indica que, de media, los españoles consultan su teléfono unas 150 veces al día, y le dedican un tiempo estimado cercano a las 3 horas y 20 minutos, desbancando así a otras pantallas tradicionales como la televisión o el ordenador, ya sea de sobremesa o portátil. Este hecho convierte al teléfono en una prolongación más del usuario, estableciéndose una suerte de identificación muy sugerente para las marcas que consigan penetrar en este espacio cada vez más personal.

\subsection{LAS APPS}

Las aplicaciones digitales (en adelante, apps) son programas diseñados para los teléfonos inteligentes que permiten una comunicación bidireccional entre la empresa y el usuario, gracias a la capacidad de personalización, geolocalización y ubicuidad de los equipos (Martínez Sáez, Martín Núñez y García Cubells, 2015).

Esta comunicación, en sintonía con la era digital actual, se desarrolla de un modo menos intrusivo que la publicidad tradicional, ya que se basa en contenidos que resultan relevantes para el usuario, quien voluntariamente accede a descargar e instalar este nuevo canal interactivo y permite que, en cierto modo, le atrapen, lo que incide en una valoración positiva de la marca que se esconde detrás.

Es necesario, nuevamente, acudir al informe de Ditrendia para poder contextualizar de manera gráfica el fenómeno que han supuesto las apps. El estudio (Ditrendia, 2016) señala que en España se descargan 3,8 millones de aplicaciones a diario y que estas son utilizadas durante el $89 \%$ del tiempo dedicado al terminal. Es decir, cada español invierte diariamente cerca de 3 horas en las 13 aplicaciones, de media, que tiene instaladas en su smartphone.

Estas aplicaciones, tal y como señalan González Romo y Contreras Espinosa (2012), son encontradas por los usuarios a través de la búsqueda o por recomendaciones de amigos y familiares, aunque también se presta atención a las valoraciones y comentarios de otros usuarios.

Por lo tanto, es necesario dotar a la aplicación de un contenido atractivo, relevante para el usuario y amigable, con el objetivo de lograr acceder a ese reducido espacio en cada terminal, pues, como 
indican Ortega, González y Pérez (2015, p. 629), nos encontramos ante «un canal complementario y probablemente sustitutivo en pocos años para el consumo en las pantallas y dispositivos tradicionales»

Siguiendo con estos autores, el catálogo de aplicaciones digitales logra «por primera vez en la historia [...] tener acceso en tiempo real al real data de su audiencia, sobre sus usos y consumos» (2015, p. 629). Son, por tanto, una potente herramienta, no solo para poner en valor su marca y sus productos, dotando a sus aplicaciones de un valor añadido para cada usuario, sino que, además, en este nuevo canal de comunicación bidireccional, obtiene de manera gratuita un valioso feedback por parte de su público potencial.

\subsection{LA EXHIBICIÓN CINEMATOGRÁFICA}

Hablar de cine es hablar de crisis, no solo en cuanto a lo económico, sino también en cuanto a lo tecnológico. La industria cinematográfica, al igual que la telefónica, vive sumida en un constante proceso de reinvención para distanciarse de sus competidores y seguir atrayendo espectadores.

De este modo, la sucesión de 24 fotogramas por segundo abrazaba las llegadas del sonido en 1927, con la película El cantor de jazz (Crosland, 1927), y del color mediada la década de los treinta, con La feria de las vanidades (Mamoulian, 1935).

Estos cambios, sin duda decisivos en la historia del séptimo arte, afectaban de manera vertical a toda la cadena de elaboración de la película, esto es, producción, distribución y exhibición.

Sin embargo, y como señala Ansola González (2003), este último sector «es el más acusado a nivel tecnológico y económico, ya que durante las tres últimas décadas ha tenido que adaptarse a las modificaciones del mercado a través de remodelaciones infraestructurales con costes muy elevados».

Bajo el paraguas del boom inmobiliario de la década de los 90 proliferaron en España los complejos multicines o multiplex, insertos en grandes centros comerciales, elevando la cifra de pantallas hasta las 4.401 en el año 2005, con más de 127 millones de espectadores (MECD, 2015).

En la actualidad, en un contexto de crisis económica global y tras el desplome en el número de espectadores -se han perdido casi 40 millones en 10 años-, «casi dos tercios del parque de salas de cine no resultan rentables» (García y Maestro, 2015, p. 77), por lo que la progresiva adaptación del sector de la exhibición a la tecnología digital y el 3D, en su lucha por diferenciarse del resto de pantallas, resulta verdaderamente complicada.

Una de las tendencias en el mercado cinematográfico es la concentración de empresas exhibidoras, rompiendo así con una «atomización» que distanciaba al mercado español del europeo, tal y como recogen García y Maestro (2015). De este modo, en los últimos años se han llevado a cabo diversas operaciones de fusión y absorción entre operadores, dando lugar a una mayor concentración empresarial, de la que ya han desaparecido actores tan importantes como Ábaco, UGC, AMC o Lusomondo.

Los movimientos corporativos no han logrado, sin embargo, paliar el principal problema que afecta a la industria cultural, y que es el imparable ascenso de la piratería o acceso ilegal a contenidos con propiedad intelectual. A pesar de la Ley de Propiedad Intelectual, en vigor desde el 1 de enero de 2015, el año pasado se consumieron en España 878 millones de contenidos vinculados a la industria cinematográfica, con un valor estimado de 6.907 millones de euros (Fernández Díaz, 2016). Es necesario en este punto recordar que los ingresos en taquilla del conjunto del territorio español ascendieron a 571 millones de euros en todo 2015 (El Confidencial, 2015), es decir, poco más de un 8\% del valor atribuido al consumo ilegal.

Además, se han producido movimientos empresariales a distinto nivel para ofrecer al espectador contenidos legales fuera de las salas cinematográficas, bien pagando por títulos o bien mediante una suscripción mensual, con lo que la ventana de explotación en cartelera se reduce drásticamente. 
Así, como señala Izquierdo Castillo (2012, p. 9), las majors norteamericanas -Warner Bros., Universal Studios, Metro Goldwing Mayer, Sony Pictures y Paramount Pictures- pusieron en funcionamiento el servicio Movielink, que ofrecía el visionado legal y en línea de sus películas. Su andadura finalizó en 2007, cuando fue adquirida por Blockbuster.

En el caso de los operadores de banda ancha en España -Movistar, Vodafone, Orange-, tal y como apuntan Videla Rodríguez, García Torre y Formoso Barro (2016), han lanzado al mercado servicios con acceso multidispositivo a una oferta audiovisual que incluye programación en directo y a la carta.

Por último, ahondando en esta multiplicidad de canales, cabe destacar el éxito de la plataforma de contenidos online Netflix, con más de 69 millones de suscriptores, implantada en más de 60 países y con una oferta que supera los 100 millones de horas de contenidos, tal y como señala la propia compañía en su perfil de YouTube.

Estos datos ponen de manifiesto una realidad, y es que el abandono de las salas de cine no se traduce necesariamente en una pérdida de interés por los contenidos audiovisuales. Este consumo, al contrario, ha crecido, y lo ha hecho fuera de los multicines, en Internet, tal y como apuntan en su estudio Deltell Escolar y García Fernández (2013, pp. 6-7).

\section{OBJETIVOS E HIPÓTESIS}

El objeto de análisis que planteamos, el sector de la exhibición cinematográfica en España, presenta un producto común, la película, por lo que «la imagen de marca es difícilmente sostenible, y responde a otros factores como la notoriedad, la comodidad o la singularidad de sus complejos» (García Santamaría, 2009, p. 7).

Atendiendo a esta particularidad, y dado que la batalla por el consumo audiovisual se está librando fuera de las salas, en la red, planteamos como objetivo general de esta investigación analizar de manera cualitativa las apps de los principales circuitos de exhibición en España, conformados por las empresas Cinesa y Yelmo.

Con esta meta en el horizonte, planteamos a continuación una serie de objetivos específicos que permiten dotar de contenido el trabajo de investigación:

- Comprobar si las aplicaciones aprovechan las prestaciones de los dispositivos móviles, a saber: usabilidad, personalización, integración multimedia, geolocalización y socialización.

- Comparar los contenidos de las aplicaciones con los alojados en las páginas web de cada exhibidor, a fin de determinar si aportan un valor añadido para el usuario.

- Examinar si las aplicaciones disponen de herramientas social data, con las que poder conocer las preferencias de su público objetivo.

Del mismo modo, y en base a la bibliografía consultada, proponemos como hipótesis principal que el planteamiento desarrollado en las apps analizadas, correspondientes a los principales exhibidores cinematográficos en España, es a todas luces insuficiente.

De manera específica, consideramos que las aplicaciones no aprovecharán todo el rendimiento que ofrecen los terminales móviles, dado el retraso habitual en la adopción de nuevas tecnologías que afecta tradicionalmente al negocio cinematográfico. 
Esperamos igualmente un resultado negativo en cuanto a la aportación de valor añadido a través de las aplicaciones, que presumiblemente replicarán los contenidos de la página web en un formato reducido.

Por último, respecto a la utilización de herramientas social data para conocer de manera detallada al usuario y ofrecerle una experiencia personalizada, consideramos, en concordancia con las premisas anteriores, que las apps analizadas no estarán dotadas de las herramientas necesarias para mantener esa conversación bidireccional con el usuario.

\section{METOdologíA de ANÁLISIS}

En el aspecto metodológico, la presente investigación parte de un enfoque analítico descriptivo, confirmado en la revisión bibliográfica, si bien queremos dejar patente que no existe unanimidad a la hora de categorizar las prestaciones de una app.

De este modo, nuestro estudio se basa en los trabajos de Crescenzi Lanna y Grané Oró (2016), que analizan el diseño visual, la adaptabilidad, la interactividad, la estructura y la navegabilidad en aplicaciones educativas; de González Romo y Contreras Espinosa (2012), enfocada a la socialización, el diseño y la exclusividad en las marcas de prestigio; Costa Sánchez (2012), centrado en la multimedialidad, la usabilidad, la hipertextualidad y la interactividad en la prensa digital; Ventura Salom (2011), que analiza la personalización, la socialización, la geolocalización y el acceso a contenidos adicionales en medios informativos; Camuñas Maroto (2013), que comprueba la interactividad de las aplicaciones de RTVE; Nozal Cantarero y González Neira (2012) sobre la usabilidad, la interactividad y los contenidos multimedia en las aplicaciones de la prensa italiana y española; o el estudio de Videla Rodríguez, García Torre y Formoso Barro (2016) en cuanto a los contenidos y la interactividad en las apps de las televisiones españolas.

Esta disparidad de criterios, lejos de generar un problema a la hora de afrontar este trabajo, supone un estímulo, ya que la amplitud de perspectivas adquiridas en los referentes consultados ha permitido confeccionar una completa ficha de análisis que dará debida cuenta de los siguientes aspectos:

\author{
Posicionamiento \\ Modelo de negocio \\ Personalización \\ Geolocalización \\ Exclusividad
}

\author{
Feedback \\ Usabilidad \\ Archivos multimedia \\ Interactividad \\ Uso online/offline
}

Estos criterios de análisis serán aplicados sobre las aplicaciones digitales de las dos principales compañías en el sector de la exhibición en España, Cinesa y Yelmo ${ }^{1}$.

Cinesa, fundada en 1958, posee a día de hoy el mayor circuito de salas de cine en España, con más de 500 pantallas repartidas en más de 40 multicines, con unas 100.000 butacas, y que acogen anualmente 17 millones de espectadores en sus más de 300 estrenos.

Yelmo, por su parte, nace en 1981, y en la actualidad cuenta con más de 400 salas localizadas en 37 centros multipantalla, y recibe anualmente a 13 millones de espectadores, lo que la sitúa en el segundo puesto de exhibidores.

\footnotetext{
${ }^{1}$ La búsqueda se ha llevado a cabo sobre el catálogo digital de Google Play Store, ya que, según Ditrendia (2016) y Kantar Media (2016), el sistema operativo Android es el más utilizado por los españoles, con más del $90 \%$ de presencia en el mercado de apps. Los datos ofrecidos pertenecen a las webs oficiales de ambas compañías, Cinesa (http://www.cinesa.es/Acerca-deCinesa) y Yelmo (http://yelmocinesb2b.es/que-es-yelmo-cines/).
} 
No es objeto de estudio en esta investigación realizar un análisis de la trayectoria de ambas empresas, por lo que recomendamos a los lectores interesados en esta materia los trabajos de García Santamaría (2009), en solitario, y junto a Maestro Espínola (2012), cuya temática versa sobre las empresas de exhibición cinematográfica nacionales.

\section{RESULTADOS DE LA INVESTIGACIÓN}

Una vez aplicado el instrumento de investigación sobre las aplicaciones para smartphone de Cinesa y Yelmo pasamos a detallar los resultados obtenidos en la siguiente tabla:

Tabla 1. Prestaciones de las apps

\begin{tabular}{|c|c|c|}
\hline CARACTERísticA $\boldsymbol{A P P}$ & CiNESA & YELMO \\
\hline 1. Posicionamiento & Entretenimiento: 118 & Entretenimiento: 92 \\
\hline & +100.000 descargas & +100.000 descargas \\
2. Feedback & $3,9 / 5$ estrellas & $3 / 5$ estrellas \\
& 4.760 votos & 1.109 votos \\
\hline 3. Modelo de negocio & Gratuito & Gratuito \\
\hline 4. Usabilidad & No & No \\
\hline 5. Personalización & Sí & No \\
\hline 6. Multimedia & Sí & Sí \\
\hline 7. Geolocalización & No & No \\
\hline 8. Interactividad & Sí & No \\
\hline 9. Exclusividad & No & No \\
\hline 10. Uso online/offline & No & . \\
\hline
\end{tabular}

Fuente: elaboración propia (datos descargados a 28/09/2016).

Como se desprende de los datos recogidos en la Tabla 1 (Prestaciones de las apps), ambas empresas han puesto los cimientos de lo que debería ser un nuevo canal de comunicación con el espectador, basado en la bidireccionalidad y la aportación de valor. Sin embargo, y salvo en la integración de contenidos multimedia, los dos casos presentan importantes lagunas que deberán ser subsanadas para lograr un mejor posicionamiento en la red y una mayor afluencia a las salas cinematográficas.

1. Si atendemos al Posicionamiento en el catálogo de la App Store, ambas empresas pertenecen a la categoría de entretenimiento, si bien su representatividad es reducida, pues ocupan las posiciones 92 (Yelmo) y 118 (Cinesa). No obstante, el comportamiento habitual del usuario es acceder a una determinada aplicación a través de la búsqueda textual. Así pues, si se introduce el término de búsqueda cine, Yelmo aparece en la posición número 7, mientras que Cinesa queda relegada a la 10, por detrás de aplicaciones asociadas a sitios web (por ejemplo, IMDB) o de juegos (Cine Quiz Trivial).

2. Respecto al Feedback en el catálogo de la App Store, las dos aplicaciones superan las 100.000 descargas, lo que demuestra un bajo impacto respecto a los más de 25 millones de espectadores que suman entre ambas. Su valoración es media/alta, aunque cabe destacar que Cinesa cuida en mayor medida su relación con el usuario, respondiendo puntualmente a las cuestiones/quejas planteadas desde el portal de descarga. Esta atención, como indican González Romo y Contreras Espinosa (2012, p. 87), puede marcar la diferencia entre el éxito y el fracaso ante una aplicación mal ejecutada.

3. En cuanto al Modelo de negocio, se trata de dos apps gratuitas que, además, incorporan sus respectivas pasarelas de pago (m-commerce) para tratar de fidelizar al cliente. Sin embargo, en ambos casos reconducen a la página web de la empresa, por lo que no funcionan de manera independiente. Este hecho podría provocar que ambos programas fueran relegados a meros espacios informativos, 
sin cumplir con su función de abrir una nueva vía de comercialización para los exhibidores cinematográficos.

4. La Usabilidad de las aplicaciones es reducida, ya que, si bien es posible navegar por los menús sin necesidad de volver a la página de inicio, no permite ampliar ni reducir la visibilidad, ni modificar los colores de la fuente y el fondo, ni incorporan un manual de instrucciones o ayuda. Estas carencias, irrelevantes para el usuario avezado, pueden alejar a potenciales clientes menos habituados a estos usos tecnológicos, así como a personas con dificultades visuales.

5. La Personalización es igualmente limitada, y en el caso de Yelmo se reduce a la identificación mediante el número de tarjeta de la cadena. Por su parte, Cinesa suma además la posibilidad de registrarse por mail o a través de Facebook. Sin embargo, ninguna de las dos permite guardar favoritos ni descargar contenidos al smartphone, con lo que se pierde la posibilidad de establecer una identificación completa del espectador.

6. La Integración de contenidos multimedia supone el mayor logro de estos canales, ya que permiten el acceso, constantemente actualizado, a la sinopsis, trailer y póster de las películas exhibidas en cartelera, así como a los horarios de las diferentes sesiones.

7. La Geolocalización se afronta desde dos enfoques diferentes: mientras Yelmo hace uso del GPS integrado en el terminal para detectar la ubicación del usuario y ofrecerle las carteleras más cercanas, Cinesa opta por la selección manual, permitiendo de igual modo acceder a las exhibiciones más próximas.

8. La Interactividad es una de las principales bazas de Cinesa, que permite crear alertas para nuevos eventos, valorar las películas y compartir los contenidos a través de las redes sociales. Por su parte, Yelmo no dispone de ninguna de estas opciones y la única posibilidad de participación del usuario se reduce a un buzón de quejas y sugerencias. En palabras de Scolari (2008, p. 173) la primera «complementa y completa la comunicación del resto de canales» [traducción del autor], mientras que la segunda carece de valor añadido para el usuario final.

9. No existe Exclusividad alguna para los usuarios de ninguna de las dos aplicaciones, ya que, en la práctica, constituyen una versión reducida de los contenidos alojados en las páginas web de ambas empresas. Desaprovechan, por tanto, la oportunidad de brindar a los usuarios una serie de contenidos que aprovechen de manera eficiente las especificidades de esta nueva ventana de comunicación (Aguado Terrón y Martínez Martínez, 2009, p. 338)

10. Las dos apps han sido diseñadas para ser utilizadas Online, ya que pierden la mayor parte de sus contenidos sin el acceso a la red (vídeos, imágenes, horarios). Sin embargo, y teniendo en cuenta que más de 20 millones de españoles se conectan a diario a Internet desde sus teléfonos móviles (Fernández Lantigua, 2016), esta condición no debe suponer ningún tipo de obstáculo para su correcto funcionamiento.

\section{CONCLUSIONES Y DISCUSIÓN}

A la luz de los resultados expuestos en las páginas anteriores y tras haber alcanzado con éxito los objetivos planteados para la presente investigación, consideramos probada la hipótesis principal del estudio, es decir, a través del análisis de variables ha quedado demostrado que las aplicaciones analizadas, correspondientes a las empresas del circuito de exhibición cinematográfica Cinesa y Yelmo, hacen un uso limitado de las potencialidades que ofrece el smartphone como nuevo canal de comunicación con el espectador.

Esta conclusión es coherente con la bibliografía analizada, a pesar de las evidentes diferencias en cuanto al material de estudio, que corresponde principalmente a medios de comunicación -prensa y 
televisión españolas-, y a las diferentes metodologías utilizadas, que van desde la observación directa hasta el análisis de contenido.

A nivel específico, en el segundo capítulo habíamos aventurado una falta de aprovechamiento de los recursos y potencialidades específicas que ofrecen los smartphones, debido al retraso endémico que afecta a la industria cinematográfica en España.

Sin embargo, este aspecto no difiere del resto del sector audiovisual, ya que, como señala Camuñas Maroto (2013, p. 16), los medios de comunicación tradicionales replican en sus aplicaciones digitales los mismos contenidos alojados en sus respectivos portales web, con un reducido margen para la socialización, la interactividad y la personalización. La única prestación que integran las diferentes apps - en este caso, de RTVE- es la ubicuidad, ya que permiten acceder a la oferta audiovisual desde un formato con mayor movilidad, como es el teléfono móvil.

Siguiendo con las plataformas televisivas, el trabajo de Videla Rodríguez, García Torre y Formoso Barro (2016, p. 566) señala de manera específica que RTVE y Atresmedia han diseñado aplicaciones alternativas -+tve y Atresmedia conecta- para favorecer la participación directa del espectador, mientras que los canales principales, junto al de Mediaset -mitele-, se sitúan en el plano de la oferta de contenidos, limitando su interactividad a la capacidad de compartir en redes sociales.

En el ámbito de los medios de comunicación impresos, Costa Sánchez (2012, p. 247) incide en la posibilidad de compartir contenidos a través de las diferentes redes, aunque la opción más habitual es el envío por correo electrónico. Además, añade, su usabilidad suele ser limitada, pues solo algunos medios escritos permiten ampliar el tamaño de sus textos e imágenes.

En un plano teórico se encuentran las conclusiones de Ventura Salom (2011, pp. 31-32), que analiza las prestaciones de las aplicaciones para smartphone de los medios de comunicación a través de encuestas sobre personas de diferentes edades. Así, señala la falta de interactividad y personalización de los contenidos como principales carencias en estas nuevas vías de comunicación, al tiempo que ensalza la enorme movilidad que permiten los nuevos dispositivos.

Respecto a la tercera hipótesis planteada, en la que señalamos la escasa aportación de valor añadido a través de las aplicaciones digitales, confirmamos que se limitan a replicar, incluso en un formato más limitado, los contenidos de la página web, por lo que no aportan nada nuevo al usuario.

Esta conclusión coincide con la alcanzada por Camuñas Maroto en su estudio de las aplicaciones del grupo RTVE (2013, p. 17), en el que apunta que «todas las noticias que se insertan en las aplicaciones para smartphone o tablet son copias de las que se encuentran en la página oficial de RTVE, no aporta este soporte ningún contenido nuevo ni exclusivo, tan solo se encuentra al mismo nivel que la página web y en la mayoría de los casos una noticia reducida».

En la misma línea se sitúa el trabajo de Nozal Cantarero y González Neira (2012, p. 646) sobre las apps de la prensa italiana y española, en el que hablan de un «volcado casi automático de contenidos»y, en muchos casos, con un tratamiento en menor profundidad que en la página web.

Sin embargo, y contradiciendo lo anterior, el análisis llevado a cabo por Videla Rodríguez, García Torre y Formoso Barro (2016, p. 566) sobre los grupos de comunicación RTVE, Atresmedia y Mediaset, revela que sus aplicaciones ofrecen contenidos extra, como imágenes no emitidas en televisión, ruedas de prensa, making of o fragmentos de programas, lo que enriquece la mera emisión de contenido en directo.

Por último, respecto a la utilización de herramientas social data para obtener un conocimiento más detallado del usuario y ofrecerle una experiencia personalizada, hemos comprobado que las apps analizadas no están debidamente dotadas de las herramientas necesarias para mantener esa conversación bidireccional con el usuario. 
No obstante, no es posible realizar esta afirmación de manera categórica, ya que se intuye una cierta intencionalidad en la aplicación de Cinesa, a través del control de las valoraciones de las películas (mediante estrellas), de las alertas ante futuros eventos, o del número de veces que se comparte un determinado contenido. Todas estas opciones pueden condicionar futuras incorporaciones a cartelera o el diseño de determinadas promociones, ya que no debemos ignorar que el fin último debe ser la compra de entradas.

Este planteamiento es compartido por Reinares Lara y Calvo Fernández (1999), que sostienen que es necesario disponer de una buena tipología de clientes a los que dirigir de manera diferenciada la publicidad sobre los servicios ofrecidos de la empresa, a fin de alcanzar su fidelización.

En esta misma línea se posicionan Sendra García, Tejerina Arreal y García Guardia (2014, p. 440), al sostener que «estos sistemas se centran en la segmentación de los mensajes en función del tipo de consumidor teniendo en cuenta dónde y cuándo adopta sus decisiones de compra permitiendo, de esta manera, conocer la atención de la audiencia y calificarla».

Salvador Benítez (2013, pp. 107-108), por su parte, recuerda que todos los productos audiovisuales «tienen un nicho en el mercado. Por eso es necesario recabar toda la información del mismo: quién consume o podría consumir el producto, perfil psicosocial (actitudes, intereses, deseos, motivaciones), contexto social y cultural, grupo social de referencia, estilo de vida, hábitos de consumo, etc.», lo que no hace sino aportar un mayor valor al estudio de la audiencia potencial, a fin de ofrecer un contenido relevante y valioso para ella.

Se trata, por tanto, de aprovechar las nuevas posibilidades que brindan las aplicaciones digitales, y establecer una relación directa y bidireccional con el espectador. El sector de la exhibición debe evolucionar de manera firme al entorno digital, como señala Calvo Herrera (2011), que debe servir de complemento a los medios tradicionales de promoción, como la prensa, la radio o la televisión.

Esta investigación ha pretendido abrir una nueva vía para el estudio académico de un sector, el de la exhibición cinematográfica, que tradicionalmente se ha mostrado receloso a encarar una apertura hacia la red. No obstante, consideramos que el correcto aprovechamiento de las posibilidades que brindan los últimos avances tecnológicos -smartphones y apps- permitirá atraer nuevamente a los espectadores a las salas.

\section{REFERENCIAS}

Aguado Terrón, J. M. y Martínez Martínez, I. (2009). Mobile Media Implicit Cultures: Towards a Characterization of Mobile Entertainment and Advertising in Digital Convergence Landscape. Observatorio Journal, 13, 336-352.

Ansola González, T (2003). Muchas sombras y pocas luces. La comercialización de las películas españolas durante la década de los noventa. ZER Revista de Estudios de Comunicación, 15, 153-173.

Calvo Herrera, C. (2011). Marketing cinematográfico off line y on line. Revista DereCom, 6, 1-7.

Camuñas Maroto, M. (2013). Diseño e interactividad en las aplicaciones de RTVE adaptadas para dispositivos móviles. En Actas del V Congreso Internacional Latina de Comunicación Social, Universidad de La Laguna, Canarias.

Costa Sánchez, C. (2012). Ciberperiodismo en el smartphone. Estudio de la multimedialidad, usabilidad, hipertextualidad e interactividad de las aplicaciones de medios nativos digitales para smartphone. Estudios Sobre El Mensaje Periodístico, 18, 243-251.

Costa Sánchez, C. (2014). Las singularidades del medio móvil: integración multimedia, personalización, geolocalización y participación. Estudio de su presencia en las apps de la prensa española. Palabra Clave, 17(3), 672-694. 
Crescenzi Lanna, L. y Grané Oró, M. (2016). Análisis del diseño interactivo de las mejores apps educativas para niños de cero a ocho años. Comunicar, 46, 77-85.

Deltell Escolar, L. y García Fernández, E. C. (2013). La promoción fílmica en el universo digital. Hacia el ocaso de la exhibición cinematográfica en España. Historia y Comunicación Social, 18 (noviembre), 203-217

Ditrendia (2016). Informe Mobile en España y en el Mundo 2016. Recuperado en 26/09/2016 de: http://www.ditrendia.es/wp-content/uploads/2016/07/Ditrendia-Informe-Mobile-enEspa $\%$ C3\%B1a-y-en-el-Mundo-2016-1.pdf

El cine en España suma 571 millones de euros y 94 millones de espectadores en 2015. (30 de diciembre de 2015). El Confidencial. Recuperado en 25/09/2016 de:

http://www.elconfidencial.com/cultura/cine/2015-12-30/el-cine-en-espana-suma-571-millonesde-euros-y-94-millones-de-espectadores-en-2015 1129043/

Fernández Díaz, E. (31 de marzo de 2016). La piratería resiste la ley. El Mundo. Recuperado en 25/09/2016 de: http://www.elmundo.es/television/2016/03/31/56fd4451268e3eda138b457f.html

Fernández Lantigua, A. (4 de abril de 2016). El móvil supera por primera vez al ordenador para acceder a Internet. El Mundo. Recuperado en 25/09/2016 de: http://www.elmundo.es/sociedad/2016/04/04/57026219e2704e90048b465e.html

García Santamaría, J. V. (2012). La reinvención de la exhibición cinematográfica: centros comerciales y nuevas audiencias de cine. ZER Revista de Estudios de Comunicación, 17(32), 107-119.

García Santamaría, J. V., Maestro Espínola, L. (2015). La exhibición cinematográfica española: una industria en recesión. adComunica, 10, 77-97.

González Romo, Z. y Contreras Espinosa, R. (2012). Apps como una posibilidad más de comunicación entre la marca y su público: un análisis basado en la valoración de los usuarios. Pensar La Publicidad. Revista Internacional De Investigaciones Publicitarias, 6(1), 81-100.

KantarMedia (2016). Kantar Worldpanel. Recuperado en 26/09/2016 de: http://es.kantar.com/tech/m\%C3\%B3vil/2016/junio-2016-cuota-de-mercado-de-smartphonesen-espa $\%$ C3\%B1a-abril-2016/

Martínez Sáez, J., Martín Núñez, M. y García Cubells, M. R. (2015). Un poco de rock \& love. Recursos creativos empleados por las marcas en la publicidad para móviles. Icono 14, 13, 76-104.

Nozal Cantarero, T. y González Neira, A. (2012). La interactividad en las aplicaciones periodísticas para iPad italianas y españolas. Estudios sobre el Mensaje Periodístico, 18, 639-648.

Reinares Lara, P. y Calvo Fernández, S. (1999). Gestión de la comunicación comercial; Madrid: MCGrawHill.

Salvador Benítez, A. (2014). Cine y medios sociales: reinventando el marketing promocional. Cuadernos De Documentación Multimedia, 24, 104-116.

Scolari, C. (2008). Online brands: Branding, possible worlds, and interactive gram-mars. Semiotica, 1, 169-178.

Ventura Salom, B. (2011). Consumo informativo en terceras pantallas: El uso de nuevos dispositivos de información en la sociedad española. En Actas del III Congreso Internacional Latina de Comunicación Social. La Laguna: Universidad La Laguna, 1-44.

Videla Rodríguez, J. J., García-Torre, M. y Formoso Barro, M. J. (2016). Contenidos e interactividad de las apps para smartphone de las televisiones españolas. Revista Latina de Comunicación Social, 71, 552-569. 